\title{
Interpretation of the Vibrational Behavior of Methyl and Substituted Methyl Radicals Perturbed by Alkali Halide Molecules
}

\author{
JEREMY BURDETT ${ }^{1}$ \\ Department of Chemistry, University of Michigan, Ann Arbor, Michigan 48104
}

The observed vibrational behavior of free and alkali halide-perturbed methyl radicals in low temperature inert matrices is rationalized on the basis of the relaxation of the molecular charge distribution of the methyl radical on vibration. It is shown that the primary interaction of an MX species with a methyl radical, is with the unoccupied, antibonding $\bar{a}_{1}{ }^{\prime}$ orbital of the latter, which, wia a perturbation treatment is linked to the electronic ground state out-of-plane bending force constant and quartic pottntial terms. The behavior of the halo substituted analogs is investigated in terms of the molecular orbital energy separation parameter, $\Delta \epsilon$, as a function of out-of-plane angle, and the number of available $\pi$-type ligand orbitals.

\section{INTRODUCTION}

The pseudo Jahn-Teller effect has been shown to be important in determining the vibrational potential function of several molecular systems ( 1 ), among them the rocking and wagging modes of $\mathrm{CH}_{2} \mathrm{~N}_{2}$ and $\mathrm{CH}_{2} \mathrm{CO}$, and the out-of-plane bending mode of $\mathrm{CH}_{3}$ itself.

The analysis of the properties of the out-of-plane bending mode of the methyl alkali halide radicals is quite similar to methyl and allows additional insight into the nature and structure of this vibrationally bizarre series.

For the ground electronic state of the methyl radical, the force constant (1) for the out-of-plane bending mode, taking into account the relaxation of the molecular electronic charge distribution, includes a sum of matrix element products over all excited electronic states ${ }^{2} A_{1}^{\prime}$, viz.,

$$
F_{22}(0)=\left\langle 0\left|\mathfrak{H}_{22}\right| 0\right\rangle-2 \sum_{m=2}^{\prime}, \quad \frac{\left.\left\langle 0\left|\mathfrak{F}_{2}\right| m\right\rangle\right|^{2}}{F_{m}^{(0)}-E_{0}^{(0)}},
$$

where we define $E_{m}^{(0)}-E_{0}^{(0)}=\Delta \epsilon(>0)$ for the lowest energy ${ }^{2} A_{2}{ }^{\prime \prime} /{ }^{2} A_{1}^{\prime}$ separation. (This out-of-plane bending mode is the only vibration of the system, predicted to have a large pseudo Jahn-Teller effect.) Under the $C_{3}$ point group, as

' Power Foundation Fellow, Magdalene College, Cambridge, England. Present address: University Chemical Laboratory, Lensfield Road, Cambridge, Englaml. 
in the $\mathrm{CH}_{3} \cdots$ MX species, the summation is over all states ${ }^{2} A_{1}$. This includes new states introduced by the presence of the alkali halide molecule. $\mathfrak{K}_{2}$ is intrinsically concerned with the methyl system and so the only significant cuntributions to $\left\langle 0\left|\mathfrak{H}_{2}\right| m\right\rangle$ will be from states $|m\rangle$ whose molecular orbital description includes large contributions from the methyl atomic orbitals. We need therefore only consider the relative magnitude of the transition density localized in the region of the carbon atom by analogy with free methyl (1).

\section{THE INTERACTION}

We consider the simplest interaction geometry of an $\mathrm{MX}$ species with the $\mathrm{CH}_{3}$ radical, namely along the threefold axis of a planar methyl radical, and consider the shift of the methyl radical energy levels in such a scheme. To verify qualitative ideas concerning this interaction we have performed a series of Extended Hückel (2) calculations. These, however, poorly reproduce the methyl energy levels and in its place, a simulated methyl radical $\left(\mathrm{CH}_{3}{ }^{\prime}\right)$ has been used. This consists of a carbon $2 p_{z}$ and carbon $2 s$ orbitals with $H_{i i}$ adjusted to fit the electronic spectral data of Herzberg (3) on the methyl $\tilde{X}^{2} A_{2}^{\prime \prime}$ and $\widetilde{B}^{2} A_{1}^{\prime}$ states, respectively. (This assumes no interaction with $M$ of the hydrogen $1 s$ orbitals). The carbon $2 p_{x, y}$ orbitals are of the wrong symmetry to interact with these orbitals and, although included, were superfluous to the analysis. $M$ and $X$ ionization potential data were used as the remaining $H_{i i}$ and taken from atomic spectral tabulations (4). The $H_{i j}$ were computed using the Wolfsberg-Helmholz relationship (5) with $K=1.75$.

$$
H_{i j}=1 / 2 K S_{i j}\left(H_{i i}+H_{j j}\right)
$$

where $S_{i j}$ is the overlap integral for a given pair of atomic orbitals $i$ and $j$. MX distances were taken from the gas phase microwave results of Honig et al. (6) and the Slater exponents were calculated in the usual manner, those for the simulated methyl radical set being taken as the neutral carbon parameters. The distance between the radical and alkali halide molecule was varied.

The range of $\mathrm{CH}_{3}{ }^{\prime}-\mathrm{MX}$ distances covers the $\mathrm{Li}-\mathrm{CH}_{3}$ separation used in two recent calculations on methyl lithium monomcr. $2.10 \AA$ was chosen by Andrews $(7)$ in his vibrational analysis, and $2.28 \AA$ was used $(8)$ in a charge-iterated Hückel investigation of the bonding situation in $\mathrm{CH}_{3} \mathrm{Li}$ monomer. Presumably, because of the increase in energy of the $\mathrm{Li} \sigma$-type orbitals due to the presence of the halogen atom, $\mathrm{CH}_{3} / \mathrm{M}$ bonding is reduced considerably in the $\mathrm{CH}_{3} / \mathrm{LiX}$ series compared to $\mathrm{CH}_{3} \mathrm{Li}$. The bond overlap population methyl $\left(a_{2}^{\prime \prime} / \mathrm{Li}\right)$ at a distance of $2.30 \AA$ for $\mathrm{CH}_{3} \cdots$ LiBr is found to be an order of magnitude smaller than the total $\mathrm{Li}-\mathrm{C}$ overlap population calculated (8) for methyl lithium monomer. At a distance of $2.0 \AA$ the methyl $a_{2}^{\prime \prime}$ coefficient in the highest occupied molecular orbital derived from carbon $p_{\varepsilon}$ /metal $\sigma$ interaction varies between 


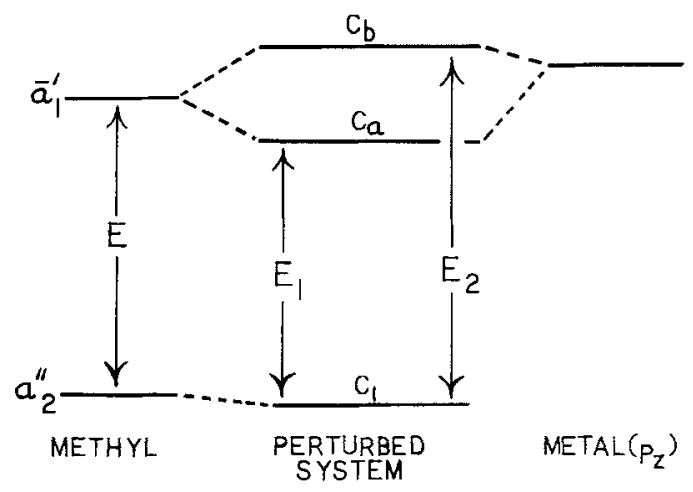

FIG. 1. Interaction scheme

0.94 and 1.00 in the $\mathrm{CH}_{3} / \mathrm{MX}$ series. For conceptual stability on the threecenter model, this orbital should contain approximately equal contributions from this methyl orbital and a halogen $\sigma$-type orbital $(9)$.

In general we find that the predominant interaction between an MX species and a planar methyl radical is with the unoccupied antibonding orbitals of the latter, the highest occupied methyl orbital $\left(p_{z}\right)$ being little affected in the perturbation. From the size of the molecular orbital coefficients in the higher unoccupied orbitals that we obtain from these calculations, the perturbation is probably best represented as the interaction of a metal $s p$ hybrid with an antibonding $\bar{a}_{1}^{\prime}$ methyl orbital. A general scheme is shown in Fig. 1.

The degree of interaction of these two orbitals will be dependant upon the energy of separation of the $M \sigma$ orbitals and the antibonding $\bar{a}_{1}^{\prime}$ methyl orbital, which are both unoccupied. The metal $p$ orbital ionization energies are smaller than the methyl parameter and increase in the order $\mathrm{K}<\mathrm{Na}<\mathrm{Li}$. The interaction energies of this orbital with the antibonding methyl orbital under consideration increases in the same order for a given $\mathrm{CH}_{3}-\mathrm{MX}$ distance, the nature and energy of the methyl $a_{2}^{\prime \prime}$ orbital being little affected in the process.

\section{VIBRATIONAL BEHAVIOR}

To reproduce a charge field of species $a_{2}^{\prime \prime}$ (under $D_{3 h}$ ), (i.e., of the same species as the out-of-plane mode) only the coefficient of the $2 p_{z}$ carbon atomic orbital in the molecular orbital derived from methyl $a_{2}^{\prime \prime}$, and the coefficient of the carbon $2 s$ orbital in the two new orbitals produced by interaction of the antibonding $\bar{a}_{1}^{\prime}$ methyl and alkali metal $\sigma$ orbital need be considered. As we have noted the transition density must be localized in the region of the carbon atom. The $2 p_{3}$ coefficient in the methyl $a_{2}{ }^{\prime \prime}$ orbital is, as suggested, only slightly affected in the perturbation.

With reference to the notation of Fig. 1, for the methyl radical itself, the pseudo 
Jahn-Teller softening term due to the presence of one close ${ }^{2} A_{1}^{\prime}$ state is;

$$
-\frac{2}{E}\left|\left\langle a_{2}^{\prime \prime}\left|\mathfrak{H}_{2}\right| \bar{a}_{1}^{\prime}\right\rangle\right|^{2}
$$

For a perturbed methyl radical the softening term is similarly

$$
-\left[\frac{c_{1}^{2} c_{a}^{2}}{E_{1}}+\frac{c_{1}^{2} c_{b}^{2}}{E_{2}}\right] \cdot 2\left|\left\langle a_{2}^{\prime \prime}\left|\mathcal{H}_{2}\right| \bar{a}_{1}^{\prime}\right\rangle\right|^{2},
$$

where $c_{1}$ is the coefficient $(\sim 1)$ of the carbon $2 p_{z}$ orbital in the molecular orbital derived from the methyl $a_{2}{ }^{\prime \prime}$ orbital and $c_{a}$ and $c_{b}$ are the coefficients of the carbon $2 s$ orbital in the two new orbitals derived from the interaction of the antibonding methyl $\tilde{a}_{1}^{\prime}$ orbital with the metal $p_{z}$ orbital, relative to that in the unperturbed methyl system.

The term in square brackets (we shall call this $K$ ) is less than that for the isolated methyl radical (see Appendix). On this basis a smaller pseudo JahnTeller softening of the methyl $a_{2}{ }^{\prime \prime}$ force constant is expected in a perturbed methyl radical than in the free system.

Identity of the Perturbing $M X$ Species. The value of this symmetric bending frequency will be dependent upon the masses of $M$ and $X$ (in general force field treatment) and also upon the electronic nature of these two atoms. In a normal coordinate analysis of this $A B_{3} C D$ system, the reduced masses of $\mathrm{M}$ and $\mathrm{X}$ do not appear in the diagonal $G$ matrix element associated with this mode but only in off-diagonal elements. (The mode is alone in its class under $D_{3 h}$ symmetry). Since the MX perturbation is small and involves little or no interaction with occupied methyl energy levels, then the vibrational coupling between the methyl modes and MX vibrations should indeed be small. For ${ }^{6} \mathrm{LiI}_{\mathrm{I}}$ and ${ }^{7} \mathrm{~L}$ iI the differences are of the same order as the experimental uncertainty, $0.1 \mathrm{~cm}^{-1}$ at $730 \mathrm{~cm}^{-1}(9)$. [Andrews (7) in his analysis of the infrared spectrum of $\mathrm{CH}_{3} \mathrm{Li}$ monomer found, however, a similar insensitivity of the symmetric bending mode to the lithium atomic mass.] Any vibrational coupling of this mode to the symmetric hydrogen stretching mode (of the same species under $C_{3 v}$ ) should also be small from the large energy separation involved. To a good approximation therefore, the perturbed methyl radical may be treated as an isolated methyl system in its vibrational behavior.

A much more important factor to be considered is the electronic nature of the $\mathrm{M}$ and $\mathrm{X}$ species. Since the ionization potentials (4) of the alkali metals $(n p)$ taken from atomic spectral data decrease in the order $\mathrm{Ii}>\mathrm{Na}>\mathrm{K}$, for a given $\mathrm{M}-\mathrm{CH}_{3}$ distance, the largest perturbation by $\mathrm{MX}$, and hence the smallest softening term should be observed for LiX, by a consideration of the value of the $K$ parameter in Eq. (2). Assuming that the "classical" force constant, defined as $\left\langle 0\left|\mathfrak{F}_{22}\right| 0\right\rangle$ remains unchanged on perturbation, (probably a good approximation since the MX interaction with the occupied methyl orbitals is small) the out-of- 
TABLE I

The Out-of-Plane Bending Frequency in Free ind Alkali HalidiPerturised Methyl Radicalis [from Refs. $(9,10)$ ]

\begin{tabular}{lcc} 
System & $\nu_{2}\left(\mathrm{~cm}^{-1}\right)$ & $\nu_{H} / \nu_{D}{ }^{\mathrm{a}}$ \\
\hline $\mathrm{CH}_{3} / \mathrm{LiBr}$ & 730 & \\
$\mathrm{CH}_{3} / \mathrm{LiI}$ & 730 & 1.288 \\
$\mathrm{CH}_{3} / \mathrm{NaBr}$ & 700 & \\
$\mathrm{CH}_{3} / \mathrm{NaI}$ & 696 & 1.305 \\
$\mathrm{CH}_{3} / \mathrm{KI}$ & 680 & 1.319 \\
$\mathrm{CH}_{3}$ & 611 & \\
\hline
\end{tabular}

a Theoretically for planar system 1.291 on harmonic model.

plane bending frequencies should be in the order $\mathrm{MI}=\mathrm{Li}>\mathrm{Na}>\mathrm{K}$, as is indeed observed. The effect of the $\mathbf{X}$ atom, removed as it is a larger distance from the radical than is the metal atom, is expected to have a relatively smaller electronic effect on the out-of-plane force constant. This must be electronic in origin since it is a much larger shift than any observable metal mass effect (Table I). (This is of course assuming that it is the $\mathrm{M}$ atom which is juxtaposed to the methyl system.)

The degree of interaction of the $\mathrm{X}$ atom with the system will depend similarly upon the ionization potential of $\mathrm{X}$. The closer the ionization potentials of $\mathrm{CH}_{3}\left(p_{3}\right)$ and X, the closer to equality their coefficients in the "nonbonding" orbital of a three-center scheme. Hence the greatest perturbation by MIX will be for $\mathrm{X}=$ iodine. For a given $\mathrm{CH}_{3} / \mathrm{MX}$ distance, the $\mathrm{CH}_{3} \cdots \mathrm{MI}$ species should therefore exhibit higher out-of-plane bending frequencies than $\mathrm{CH}_{3} \cdots \mathrm{MBr}$ species. Coupled with the data for $\mathrm{NaX}$ and $\mathrm{LiX}$ perturbed species, we conclude that the $\mathrm{CH}_{3} / \mathrm{MI}$ distance is probably greater than the $\mathrm{CH}_{3} / \mathrm{MBr}$ separation for $\mathrm{M}=\mathrm{Nat}$, li. It is readily apparent, from consideration of Van der Waals' radii that the $\mathrm{CH}_{3}$ radical has to occupy a substitutional site in an argon lattice for it to be able to freely rotate, according to the data of Milligan and Jacox (10). Due to the discrepancy in radii between the halogens and the alkali metals, sodium and lithium, it is to be expected that the position in a matrix of an MX species may well be primarily determined by the halogen size. Now the Van der Waals' radius of argon is very similar to that of bromine, and hence an $\mathrm{MBr}$ species should fit into at substitutional site next to $\mathrm{CH}_{3}$ with ease. Iodine however, has a larger radius than argon and an MI species should considerably perturb the lattice in a substitutional site. One way to relieve this stress is to slightly displace the iodine atom (and indeed the entire MI unit) further away from the $\mathrm{CH}_{3}$ site, as can readily be seen on a model.

Quartic Potential Terms. The quartic terms in the vibrational potential expansion of $S_{2}$ (the odd powers are forbidden by symmetry for the planar geometry of the isolated system) are another feature approachable by the model. It has 
previously been shown (1) that the sign and magnitude of the coefficient governing the contribution to $S_{2}{ }^{4}$ in the vibrational potential energy expansion for the methyl radical was determined by the magnitude of the second order softening term, contributed by the relaxation of the electronic charge distribution. For small values of this parameter, the sign was negative, leading to a positive anharmonicity. However, as the pseudo Jahn-Teller softening increased, the value of the coefficient became positive, and was larger, the greater the softening parameter $\alpha^{2} / \epsilon$. This analysis can be applied directly to the present case. The pseudo Jahn-Teller vibrational softening is smallest for LiX and this is manifest in a high vibrational frequency and a negative quartic term (11) of $-1 \times 10_{6}^{2}$ $\mathrm{dyn} / \mathrm{cm}^{3}$. For $\mathrm{NaX}$ and $\mathrm{KX}$, the out-of-plane mode occurs at a lower frequency, and is associated with a negative anharmonicity, which is more pronounced for $\mathrm{CH}_{3}$ than when $\mathrm{NaX}$ is the perturbing molecule. ( $\mathrm{In} \mathrm{CH}_{3}$ the ration of $\nu_{H} / \nu_{D}$ is greater than in $\mathrm{CH}_{3} \mathrm{Li}$ monomer ( $(7)$ for $\nu_{2}$.)

With the two state approximation (1) the quartic term for the methyl radical can be written via fourth order perturbation theory ( 1 ) as,

$$
\begin{aligned}
S_{2}{ }^{4}\left[\frac{1}{24}\left\langle 0\left|\mathfrak{F}_{2222}\right| 0\right\rangle-\frac{1}{3} \frac{\left\langle 0\left|\mathfrak{K C}_{2}\right| n\right\rangle\left\langle n\left|\mathfrak{F}_{222}\right| 0\right\rangle}{E_{n}^{(0)}-E_{0}^{(0)}}\right. & \\
& \left.+\frac{1}{2} \frac{\left|\left\langle 0\left|\mathfrak{F C}_{2}\right| n\right\rangle\right|{ }^{2}\left\langle n\left|\mathfrak{F}_{22}\right| n\right\rangle}{\left(E_{n}^{(0)}-E_{0}^{(0)}\right)^{2}}\right],
\end{aligned}
$$

a simplified form of the more general expression given in Ref. (1).

The second term contains the third derivative of the Hamiltonian and may be small [it was neglected in previous discussion (1)]. In any case this is combined with the first term and Eq. (3) rewritten as:

$$
\frac{1}{24} S_{2}{ }^{4}\left[N+12 \frac{\left|\left\langle 0\left|\mathfrak{K}_{2}\right| n\right\rangle\right|^{2}}{(\Delta \epsilon)^{2}}\left\langle n\left|\mathfrak{K}_{22}\right| n\right\rangle\right],
$$

where $N<0$. $\left(\left\langle 0\left|\mathscr{F}_{2222}\right| 0\right\rangle\right.$ is expected to be negative for a mode of this sort $)$. For a large pseudo Jahn-Teller effect the second term in this expression is large and a negative anharmonicity, as in the methyl radical will be observed.

For the $\mathrm{CH}_{3} / \mathrm{MX}$ case, where there are now two excited states $n_{1}$ and $n_{2}$ to be considered, the corresponding expression is:

$$
\begin{aligned}
\frac{1}{24} S_{2}{ }^{4}\left[N^{\prime}+12\left(\frac{\left|\left\langle 0\left|\mathfrak{K}_{2}\right| n_{1}\right\rangle\right|^{2}}{\left(E_{n_{1}}-E_{0}\right)^{2}}+\frac{\left|\left\langle 0\left|\mathfrak{K}_{2}\right| n_{2}\right\rangle\right|^{2}}{\left(E_{n_{2}}-E_{0}\right)^{2}}\right.\right. \\
\left.\left.+2 \frac{\left\langle 0\left|\mathfrak{K}_{2}\right| n_{1}\right\rangle\left\langle n_{2}\left|\mathfrak{K}_{2}\right| 0\right\rangle}{\left(E_{n_{1}}-E_{0}\right)\left(E_{n_{2}}-E_{0}\right)}\right)\left\langle n_{1}\left|\mathfrak{K}_{22}\right| n_{2}\right\rangle\right],
\end{aligned}
$$


or in the same nomenclature used earlier:

$$
\begin{aligned}
& \frac{1}{24} S_{2}{ }^{4}\left[N^{\prime}+12\left(\frac{c_{1}{ }^{2} c_{a}{ }^{2}}{E_{1}{ }^{2}}+\frac{c_{1}{ }^{2} e_{b}{ }^{2}}{E_{2}{ }^{2}}+\frac{2 c_{1}{ }^{2} c_{a} c_{b}}{E_{1} E_{2}}\right)\right. \\
&\left.\cdot\left|\left\langle a_{2}{ }^{\prime \prime} \mathcal{F C}_{2} \mid \bar{a}_{1}^{\prime}\right\rangle\right|^{2}\left\langle\bar{a}_{1}^{\prime}\left|\mathcal{F C}_{22}\right| \bar{a}_{1}{ }^{\prime}\right\rangle\right],
\end{aligned}
$$

to be compared with the methyl case itself

$$
\frac{1}{24} S_{2}^{4}\left[N+12 \frac{1}{E^{2}}\left|\left\langle a_{2}^{\prime \prime}\left|\mathfrak{K}_{2}\right| \bar{a}_{1}^{\prime}\right\rangle\right|^{2}\left\langle\bar{a}_{1}^{\prime}\left|\mathfrak{F}_{22}\right| a_{1}^{\prime}\right\rangle\right]
$$

We shall call the term

$$
\left(\frac{c_{1} c_{a}}{E_{1}}+\frac{c_{1} c_{b}}{E_{2}}\right)^{2}
$$

$K^{\prime}$. This will become smaller (see Appendix), the greater the interaction of the MX system with the methyl radical and will, therefore, decrease in the order $\mathrm{CH}_{3}>\mathrm{KX}>\mathrm{NaX}>\mathrm{LiX}$. The extent of the observed negative anharmonicity also drops in this order, until for $\mathrm{LiX}$ a small positive anharmonicity is observed, in good qualitative agreement with Eq. (6).

\section{SUBSTITUTED METHYL RADICALS}

In a 25-electron species $A X_{3}$, where the ligands have accessible $p$ orbitals, $\pi$ bonding in the planar configuration will raise the energy of the $a_{2}^{\prime \prime}$ orbital. Also the increase in ligand electronegativity (from $H$ to halogen) will shift the $\sigma$ framework to lower energy. This combination of factors decreases the $a_{2}^{\prime \prime} / \bar{a}_{1^{-}}$energy separation relative to $A H_{3}$ and will tend to increase the pseudo Jahn' Teller softening in this $a_{2}^{\prime \prime}$ symmetry coordinate such that the radical may be bent from planar (12).

We now therefore investigate how the magnitude of the pseudo Jahn-Teller effect in the symmetric bending mode changes (a) as a function of out-of-plane angle, and (b) as a function of the degree of halogen substitution.

(a) As the out-of-plane angle increases, the energy separation, $\Delta \epsilon$, occurring in the pseudo Jahn-Teller recipe of Eq. (1) rapidly increases by consideration of Walsh's scheme (13). Similarly the amount of $p$ character localized on the carbon atom decreases due to the formation of (at the tetrahedral geometry) an $s p{ }^{3}$ hybrid, containing the unpaired electron.

(b) As the degree of halogen substitution increases, the orbital containing the unpaired electron becomes less localized on the carbon atom, due to the formation of a multicenter molecular orbital involving $\pi$-type orbitals on the ligands.

Since experimental study (14) indicates that the angle from planarity increases with halogen substitution in this series, from effects (a) and (b) above, the 
magnitude of the $\Delta \epsilon$ term increases, and the value of the matrix element in the pseudo Jahn-Teller term decreases with an increasing degree of halogen substitution. Hence the importance of the pseudo Jahn-Teller effect should drop off fairly rapidly in the series $\mathrm{CH}_{3}, \mathrm{CH}_{2} \mathrm{X}, \mathrm{CHX}_{2}, \mathrm{CX}_{3}$, and should be associated with a similar decrease in sensitivity to MIX perturbation.

For $\mathrm{CX}_{3}$ systems, no evidence of $\mathrm{MX}$ perturbation has been observed, although $\nu_{2}$, the symmetric bending has not been detected in studies on these species. For $\mathrm{CCl}_{3}$ and $\mathrm{CCl}_{2} \mathrm{Br}$, the vibrational spectra of thermally produced radicals (15) compares well, within experimental limits, to those obtained via alkali metal halogen abstraction (16). Correspondingly, no vibrational anomalies of the type encountered with $\mathrm{CH}_{3}$ have been reported either for $\mathrm{CCl}_{3}$ (16) or $\mathrm{CBr}_{3}$ (17).

However for $\mathrm{CHX}_{2}$ systems, there are indications of $\mathrm{MX}$ perturbation from infrared evidence. Andrews suggests that $\mathrm{MX}$ perturbation occurs with $\mathrm{CHBr}_{2}$ (18) and $\mathrm{CHCl}_{2}(19)$, and $\mathrm{CHF}_{2}$ (20).

Jacox and Milligan's report (21) of the stabilization of $\mathrm{CH}_{2} \mathrm{~F}$ in an inert matrix is concerned with vibrational anomalies in a hydrogen bending mode, which, at present, are not fully investigated. [Similar observations have been made on $\mathrm{CH}_{2} \mathrm{~N}_{2}$ and $\mathrm{CH}_{2} \mathrm{CO}$, and have been rationalized on the basis of the electron relaxation effect (1).] This radical is suggested (14) to have a deviation from planar of less than $5^{\circ}$ from ${ }^{13} \mathrm{C}$ spin resonance splittings, and may therefore be expected to have a moderate pseudo Jahn-Teller effect in $S_{z}$.

Hence vibrational anomalies, and susceptibility to MX perturbation are facets of the properties of this esoteric series of radicals amenable to interpretation within the pseudo Jahn-Teller formalism.

\section{APPENDIX}

In Eq. 2 we need to show that the term $K=c_{1}{ }^{2}\left[\left(c_{a}{ }^{2} / E_{1}\right)+\left(c_{b}{ }^{2} / E_{2}\right)\right]$ is smaller than the corresponding term for the isolated methyl radical.

If we consider two interacting orbitals 1 and 2 then the secular equations, neglecting differential overlap are:

$$
\left(\begin{array}{ll}
H_{11}-\epsilon & H_{12} \\
H_{12} & H_{22}-\epsilon
\end{array}\right)\left(\begin{array}{l}
c_{1} \\
c_{2}
\end{array}\right)=\left(\begin{array}{l}
0 \\
0
\end{array}\right) .
$$

The new orbital energies by solution of the secular determinant are:

$$
\epsilon_{i}=\frac{H_{11}+H_{22}}{2} \pm\left(\left(\frac{H_{11}-H_{22}}{2}\right)^{2}+H_{12}^{2}\right)^{1 / 2}
$$

the coefficient of orbital 1 in the lower molecular orbital (derived mainly from 1 ) is

$$
c_{a}^{2}=\frac{H_{12}^{2}}{\left(H_{11}-\epsilon_{1}\right)^{2}+H_{12}^{2}}
$$

and the coefficient of orbital 1 in the upper molecular orbital (derived mainly 
from 2 ) is

$$
c_{b}^{2}=\frac{\left(H_{22}-\epsilon_{2}\right)^{2}}{H_{12}^{2}-\left(H_{22}^{2}-\epsilon_{2}\right)^{2}}
$$

All that is now required is evaluation of the parameter $K$ by substitution.

In the zero overlap approximation

$$
\left|H_{22}-\epsilon_{2}\right|=\left|H_{11}-\epsilon_{1}\right|,
$$

and

$$
\boldsymbol{\epsilon}_{i}=\phi \pm\left(\psi^{2}+H_{12}^{2}\right)^{1 / 2}
$$

where $\phi=1 / 2\left(H_{11}+H_{12}\right)$ and $\psi=1 / 2\left(H_{11}-H_{12}\right)$. We now identify orbital 1 as the methyl $\bar{a}_{1}^{\prime}$ orbital and orbital 2 as the MX $\sigma$-type orbital. $\epsilon$ is the ionization potential of the free methyl $a_{2}^{\prime \prime}$ electron. By substitution in equation '2 we arrive at the result for $K$,

$$
K=c_{1}^{2} \cdot \frac{\phi-\psi-\epsilon}{(\phi-\epsilon)^{2}-\left(\psi^{2}+H_{12}^{2}\right)},
$$

where $\epsilon_{1}-\epsilon=E_{1}$ and $\epsilon_{2}-\epsilon=E_{2}$. Substitution for $\phi, \psi$ and using the approximation for $H_{12}$,

$$
H_{12}=1 / 2 S\left(H_{11}+H_{12}\right)
$$

we find that

$$
\begin{aligned}
K & =c_{1}^{2} \frac{H_{22}-\epsilon}{\left(H_{11}-\epsilon\right)\left(H_{22}-\epsilon\right)+\left(1-S^{2}\right)\left(\frac{H_{11}-H_{22}}{2}\right)^{2}} \\
& =c_{1}^{2} \frac{1}{\left(H_{11}-\epsilon\right)+\frac{1-S^{2}}{H_{22}-\epsilon} \cdot\left(\frac{H_{11}-H_{22}}{2}\right)^{2}}
\end{aligned}
$$

now $c_{1}^{2}<1$ since the $a_{2}^{\prime \prime}$ orbital is slightly depressed, and the parameter

$$
\left(\frac{1-S^{2}}{H_{22}-\epsilon}\right)\left(\frac{H_{11}-H_{22}}{2}\right)^{2}>0 .
$$

Hence $K<1 /\left(H_{11}-\epsilon\right)=1 / E$ the corresponding term for the isolated methyl radical.

The effect of including overlap in the above calculation tends to depress the value of $K$ even further. $\left|H_{11}-\epsilon_{1}\right|$ becomes smaller by a factor of $(1+S)^{-1}$. Both energy level changes are in the same direction and produce smaller values of $\left(E_{1}\right)^{-1}$ and $\left(E_{2}\right)^{-1}$ than in the PNDO approximation. By similar arguments 
the value of

$$
K^{\prime}=c_{1}^{2}\left(\frac{c_{a}}{E_{1}}+\frac{c_{b}}{E_{2}}\right)^{2}
$$

the anharmonicity parameter, is less than $E^{-2}$.

\section{ACKNOWLEDGMENTS}

The author is grateful to Dr. L-S. Su of this department for the use of the modified Extended Hückel program, and to E. I. duPont Ine. for support.

RECEIVED: February 14, 1970

\section{REFERENCES}

1. J. K. Burdett, J. Chem. Phys. 52, 2983 (1970).

2. The computer program used is essentially the original one used by R. Hofrman $(J$. Chem. Phys. 39, 1397 (1963)] and modified by Dr. L-S. Su of the Department of Chemistry at the University of Michigan. Calculations were performed on the University of Miehigan's IBM 360/67 computer.

3. G. Herzberg, Proc. Roy. Soc., Ser. A 262, 291 (1961).

4. C. E. Moone, "Atomic Energy Levels," Vols. 1, 2, and 3. Nat. Bur. Stand. U.S. Circ. $467,1948,1952,1958$.

5. M. Wolfsberg and L. Helmholz, J. Amer. Chem. Soc. 20, 837 (1952).

6. A. Hornig, M. Mandel, M. L. Stitch, And C. H. Townes, Phys. Rev. 96, 629 (1954).

\%. L. ANdrews, J. Chem. Phys. 47, 4834 (1967).

8. A. H. Cowley and W. D. White, J. Amer. Chem. Soc. 91, 34 (1969).

9. L. Y. Tan and G. C. Pimentel, J. Chem. Phys. 48, 5202 (1968).

10. D. E. Milligan and M. E. Jacox, $J$. Chem. Phys. 47, 5146 (1967).

11. L. Andrews and G. C. Pimentel, J. Chem. Phys. 47, 3637 (1967).

12. For a fuller discussion of these ideas see L. S. B.ARTELL, J. Chem. Educ. 45, 754 (1968).

13. A. D. Walsh, J. Chem. Soc. 1953, 2296.

14. R. W. Fessenden and R. H. Schuler, J. Chem. Phys. 43, 2704 (1965).

15. J. H. Current and J. K. Burdett, J. Phys. Chem. 73, 3504 (1969).

16. L. Andrews, J. Chem. Phys. 48, 972 (1968).

17. L. Andrews and T. G. Carver, J. Chem. Phys. 49, 896 (1968).

18. T. G. Carver and L. Andrews, J. Chem. Phys. 60, 4223 (1969).

19. T. G. Carver and L. Andrews, J. Chem. Phys. 50, 4235 (1969).

20. T. G. Carver and L. Andrews, J. Chem. Phys. 50, 5100 (1969).

21. M. E. Jacox And D. E. Mildigan, J. Chem. Phys., 50, 2352 (1969).

\section{ADDENDUM}

Snelson (1) has recently obtained the infrared spectrum of the free methyl radical in solid neon by the low-pressure pyrolysis of dimethyl mercury and of methyl iodide. Both infrared active $e^{\prime}$ modes (one stretching and one bending) were detected in addition to $\nu_{2}\left(a_{2}^{\prime \prime}\right)$. Good agreement between observed and calculated frequencies for $\mathrm{CH}_{3}$ and $\mathrm{CD}_{3}$ was obtained for $\nu_{3}$ and $\nu_{4}$. This lends support to the general idea that only the out-of-plane bending mode of this system experiences any serious effects of anharmonicity. It is also of interest to note that 